\title{
Neurolymphomatosis: A Rare Cause of Multiple Mononeuropathy
}

\author{
Gayathri Petluri'1, Manoj Kumar Goyal' ${ }^{1}$ Veenu Singla², Bhagwant Rai Mittal ${ }^{3}$, Uma Nahar4, \\ Manish Modi ${ }^{*}$, Nalini Gupta ${ }^{5}$, Dheeraj Khurana ${ }^{1}$ \\ ${ }^{1}$ Department of Neurology, Post Graduate Institute of Medical Education \& Research (PGIMER), \\ Chandigarh, India \\ ${ }^{2}$ Department of Radiodiagnosis, Post Graduate Institute of Medical Education \& Research (PGIMER), \\ Chandigarh, India \\ ${ }^{3}$ Department of Nuclear Medicine and PET, Post Graduate Institute of Medical Education \& Research \\ (PGIMER), Chandigarh, India \\ ${ }^{4}$ Department of Histopathology, Post Graduate Institute of Medical Education \& Research (PGIMER), \\ Chandigarh, India \\ ${ }^{5}$ Department of Cytology and Gynecological Pathology, Postgraduate Institute of Medical Education and \\ Research (PGIMER), Chandigarh, India \\ Email: gayathri_petluri@yahoo.com, goyal_mk@yahoo.com, modipgi@gmail.com, brmittal@yahoo.com, \\ umasakia@gmail.com, ${ }^{*}$ modim72@yahoo.com, nalini203@gmail.com, dherajk@yahoo.com,
}

Received 1 March 2014; revised 1 April 2014; accepted 8 April 2014

Copyright (C) 2014 by authors and Scientific Research Publishing Inc.

This work is licensed under the Creative Commons Attribution International License (CC BY). http://creativecommons.org/licenses/by/4.0/

(c) (i) Open Access

\section{Abstract}

Neurolymphomatosis, defined as invasion of cranial nerves and peripheral nerve roots, plexus or nerves by Non Hodgkin's Lymphoma is a very rare clinical entity. We describe a case of 69 years old gentleman, who presented to us with asymmetric, painful sensorimotor polyneuropathy. He was admitted with 2 months history of dry cough, constitutional symptoms, paraesthesias on right side of face along with painful asymmetrical quadriparesis. Nerve conduction studies were suggestive of asymmetrical sensorimotor axonal and demyelinating neuropathy. Cerebrospinal fluid analysis revealed mild pleocytosis with raised protein. FDG-PET showed intense uptake in both adrenals, abdominal lymph nodes, sacral nerve roots and brachial plexus. Fine needle aspiration cytology of adrenal mass revealed evidence of diffuse large B cell Non Hodgkin's Lymphoma. As patient succumbed to illness, an autopsy was done, which revealed diffuse large B cell lymphoma involving adrenals, brachial and lumbosacral plexuses. Our report provides important insights into a rare cause of painful demyelinating multiple mononeuropathy and emphasises on increasing role and diagnostic utility of PET imaging in evaluation of patients presenting with multiple mononeuropathy, especially with regards to paraneoplastic or neoplastic causes such as lymphoma.

"Corresponding author. 


\section{Keywords}

\section{Neurolymphomatosis, FDG PET Scan, Multiple Mononeuropathy, CIDP}

\section{Introduction}

Lymphoma can affect any part of the nervous system at any stage of the disease [1]. Peripheral nervous system is frequently involved in lymphoma and can manifest as axonopathy or demyelinating neuropathy, at times mimicking chronic inflammatory demyelinating neuropathy (CIDP). Broadly lymphoma can cause neuropathy either through direct invasion (Neurolymphomatosis) or as a paraneoplastic manifestation [2]. Neurolymphomatosis (NL) is a rare disorder characterized by lymphomatous infiltration of peripheral nervous system or cranial nerves. With availability of new diagnostic tools especially 18-Flourodeoxyglucose positron emission tomography (FDG-PET), this condition is being recognized more often [2]. Recently we came across a case of NL which is being presented here.

\section{Case Report}

This 69 years old gentleman presented to us with two month history of shooting pains in bilateral gluteal regions and back of thigh followed one month later by pin pricking paresthesias in both upper limbs and right half of face. Subsequently he developed asymmetrical quadriparesis (both distal and proximal, legs $>$ arms) without any bladder or bowel involvement. He also complained of low grade fever, dry cough, orthopnoea and significant loss of weight and appetite. He was evaluated elsewhere, where he was diagnosed as a case of chronic inflammatory demyelinating radiculoneuropathy (based on nerve conduction studies and clinical features) and was given a course of intravenous immunoglobulin. However as he continued to deteriorate he was referred to our tertiary care centre. His systemic examination at admission was unremarkable except for paradoxical breathing. Neurological examination revealed wasting and hypotonia in all four limbs, quadriparesis (both proximal and distal, left upper limb more than right and right lower limb more than left), absent deep tendon reflexes, along with patchy sensory loss involving all modalities in all 4 limbs and sensory loss on right side of face as well. Based on above clinical picture, clinical possibility of multiple mononeuropathy was considered and he was evaluated in detail for the underlying causes. Nerve conduction studies revealed motor-sensory axonal polyneuropathy. Detailed laboratory work up including hemogram, liver, renal and thyroid function tests and serum electrolytes including calcium was normal. Cerebrospinal fluid (CSF) analysis showed 60 cells (all lymphocytes), raised protein $(79 \mathrm{mg} / \mathrm{dl})$ and normal sugar $(88 \mathrm{mg} / \mathrm{dl})$. Malignant cytology was non-contributory. Detailed vasculitic work up (ANA, ANCA, serum cryoglobulins etc.) was negative. Contrast enhanced computed tomography (CECT) of chest and abdomen showed bilateral adrenal masses with patchy consolidation of right lung. Flourodeoxyglucose positron emission tomography (FDG-PET) showed intense uptake in both adrenals, abdominal lymph nodes, sacral nerve roots and brachial plexus (Figure 1).

Fine needle aspiration cytology (FNAC) of adrenal mass revealed evidence of diffuse large B cell Non Hodgkin's Lymphoma (NHL). Bone marrow examination was normal. The diagnosis considered were NL/ multiple mononeuropathy as a paraneoplastic manifestation of NHL. However in view of FNAC and PET findings, NL was considered much more likely and he was planned for further treatment. However, during the hospital stay he developed respiratory failure requiring mechanical ventilation followed by sepsis. He was given antibiotic as per culture and sensitivity results along with aggressive supportive care including mechanical ventilation. But he continued to deteriorate and succumbed to his illness about 4 weeks after admission. An autopsy was carried out which revealed evidence of lymphomatous infiltration of brachial and lumbosacral plexus. Final autopsy diagnosis was diffuse large B cell lymphoma involving adrenals as well as brachial and lumbosacral plexuses (Figure 2).

\section{Discussion}

Neurolymphomatosis (NL) is defined as invasion of cranial nerves and peripheral nerve roots, plexus or nerves by NHL. Peripheral nervous system (PNS) involvement is usually secondary to spread from systemic and only 


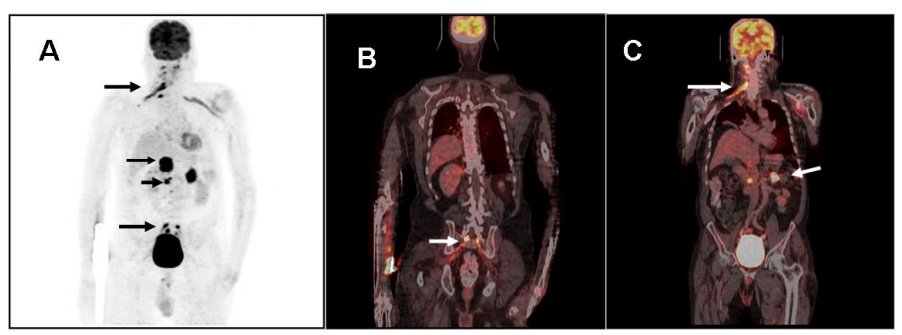

Figure 1. 18-FDG PET CT showing (A) FDG avidity in bilateral enlarged adrenals with abdominal lymph nodes (arrow heads) and Right brachial plexus and sacral nerve roots(arrows) (B) Intense FDG uptake in nerve roots (S1-S3) in sacral foramina; (C) Intense FDG uptake in adrenals and in right brachial plexus.

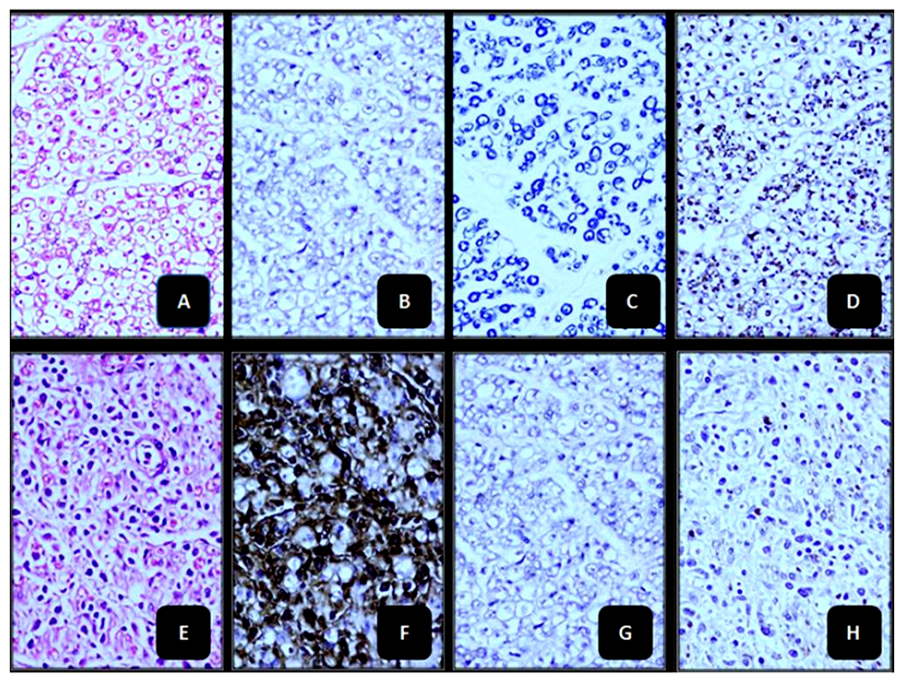

Figure 2. Panel $(\mathrm{A})-(\mathrm{H})$ (cross section of nerve bundles $\times 400$ Magnification). (A), (B), (C) \& (D)—not infiltrated by DLBCL; (E), (F), (G) \& $(\mathrm{H})$ - infiltrated by DLBCL.

rarely it is primary as seen in our patient [2]. The relative incidence of NL is estimated to be about 3\% of newly diagnosed NHL cases [3]. NL usually presents in four ways: 1) painful polyneuropathy or polyradiculopathy (most common; usually lumbosacral or cauda equina roots, rarely cervical or thoracic; usually subacute to chronic, rarely acute like Guillain-Barre syndrome), 2) cranial neuropathy (20\%; most common facial nerve followed by abducens, oculomotor and trigeminal nerve involvement), 3) painless neuropathy (sensory before motor) and 4) peripheral mononeuropathy (usually sciatic, rarely median, radial or intercostal nerve involvement). Rarely, clinical picture simulates plexus involvement. However, in one series, the commonest clinical presentation was of multiple mononeuropathy. The clinical course is either progressive or relapsing remitting. Whatsoever the initial site, NL ultimately diffusely infiltrates peripheral neural structures as well as CSF and the substance of brain and spinal cord [3].

The diagnosis of NL rests on clinical information, neuroimaging data [Magnetic resonance imaging (MRI) and FDG-PET] and morphological data obtained from either of CSF, neural or non-neural tissues. MRI reveals enlargement and enhancement of nerves, roots or plexus. FDG-PET is a useful adjunct to the diagnosis and is most sensitive and specific tool currently available for diagnosis of NL [4]-[7]. It may be positive even when all other modalities fail to yield the diagnosis as was in our case [5]. Tissue diagnosis remains the gold standard and there is tumor cell infiltration of endoneurium and perineurium. CSF analysis may reveal malignant cells in $20 \%$ - $40 \%$ of cases. In our case diagnosis of neurolymphomatosis was strongly suggested by FDG-PET findings during life and was confirmed by FNAC from adrenal mass. Autopsy done after demise of the patient confirmed the diagnosis to be NL. 
For NL, treatment is same as for CNS lymphoma and includes systemic chemotherapy alone or combined with intrathecal chemotherapy or external beam radiotherapy. With initial treatment, there is improvement in about $50 \%$, disease stabilization in about $25 \%$ and worsening in rest. However the overall response rate is poor with median survival being 10 months from initial diagnosis.

\section{Conclusion}

Our case further highlights some of the facts about this rare disorder. Neurolymphomatosis (NL), a rare manifestation of lymphoma, can precede the diagnosis of lymphoma. It also highlights difficulties in the diagnosis of this condition as detailed work-up done elsewhere (including CECT chest and abdomen) about 1 month before admission to our hospital was noncontributory. Finally it stresses on need for high index of suspicion in the setting of a painful demyelinating neuropathy. Misdiagnosis of neurolymphomatosis as chronic inflammatory demyelinating polyneuropathy (CIDP) is frequent especially in the presence of demyelinating pattern on electrophysiology. The possibility of the concomitant lymphoma should be considered in the setting of CIDP, if the patient presents with severe pain along with weakness. Moreover, it emphasizes on importance of FDG-PET in diagnosis of neurolymphomatosis. FDG-PET scan is considered to be highly sensitive in diagnosis and should be performed in all suspected cases so that appropriate management can be started at the earliest.

\section{References}

[1] Giglio, P. and Gilbert, M.R. (2006) Neurologic Complications of Non-Hodgkin’s Lymphoma. Current Hematologic Malignancy Reports, 1, 214-219. http://dx.doi.org/10.1007/s11899-006-0002-y

[2] Baehring, J.M. and Batchelor, T.T. (2012) Diagnosis and Management of Neurolymphomatosis. Cancer Journal, 18, 463-468. http://dx.doi.org/10.1097/PPO.0b013e31826c5ad5

[3] Gan, H.K., Azad, A., Cher, L. and Mitchell, P.L. (2010) Neurolymphomatosis: Diagnosis, Management and Outcomes in Patients Treated with Rituximab. Neuro-Oncology, 12, 212-215. http://dx.doi.org/10.1093/neuonc/nop021

[4] Tomita, M., Koike, H., Kawagashira, Y., Iijima, M., et al. (2013) Clincopathological Features of Neuropathy Associated with Lymphoma. Brain, 136, 2563-2578. http://dx.doi.org/10.1093/brain/awt193

[5] Salm, L.P., Van der Hiel, B. and Stokkel, M.P. (2012) Increasing Importance of 18F-FDG PET in the Diagnosis of Neurolymphomatosis. Nuclear Medicine Communications, 33, 907-916. http://dx.doi.org/10.1097/MNM.0b013e3283561881

[6] Suga, K., Yasuhiko, K., Matsunaga, N., Yujiri, T., Nakazora, T. and Ariyoshi, K. (2011) F-18 FDG PET/CT Findings of a Case of Sacral Nerve Root Neurolymphomatosis That Occurred during Chemotherapy. Clinical Nuclear Medicine, 36, 73-76. http://dx.doi.org/10.1097/RLU.0b013e3181f4a03f

[7] Cheson, B.D. (2011) Role of Functional Imaging in the Management of Lymphoma. Journal of Clinical Oncology, 29, 1844-1854. http://dx.doi.org/10.1200/JCO.2010.32.5225 